\title{
Sleep Quality and Sexual Function in Patients Under Methadone Maintenance Treatment
}

\author{
Ali Kheradmand,' Zahra Amini Ranjbar, Zahra Zeynali, ${ }^{2}$ Abdol Reza Sabahy, ${ }^{3}$ and Nouzar \\ Nakhaee ${ }^{3, *}$ \\ ${ }^{1}$ Department of Psychiatry, Behavioral Sciences Research Center, Taleghani Hospital, Research Development Committee, Shahid Beheshti University of Medical Sciences, Tehran, \\ IR Iran \\ ${ }_{3}^{2}$ Research Center for Social Determinants of Health, Institute of Futures Studies in Health, Kerman University of Medical Sciences, Kerman, IR Iran \\ ${ }^{3}$ Neuroscience Research Center, Institute of Neuropharmacology, Kerman University of Medical Sciences, Kerman, IR Iran \\ *Corresponding author: Nouzar Nakhaee, Neuroscience Research Center, Institute of Neuropharmacology, Kerman University of Medical Sciences, Kerman, IR Iran. Tel: +98-3432264196, \\ Fax:+98-3432264198, E-mail: nakhaeen@kmu.ac.ir; nakhaeen@yahoo.com
}

Received 2014 September 11; Revised 2015 February 2; Accepted 2015 February 15.

\begin{abstract}
Background: Methadone maintenance has remained the main modality of treatment for opioid dependent subjects. Side effects of methadone treatment may be potential obstacles to its continuation. Sleep quality and sexual function are two culture-based concerns, directly related to patients' compliance with methadone maintenance treatment(MMT) program.

Objectives: This research was conducted to examine the frequency of sleep disparity and sexual dysfunction in patients under MMT referring to MMT clinics of Kerman, Iran.

Patients and Methods: In this cross-sectional study, 198 adult subjects under MMT for more than 6 months were enrolled. Measurement tool consisted of Pittsburgh sleep quality index (PSQI), Arizona sexual experience scale (ASEX), the 12-item general health questionnaire (GHQ-12), and a demographic questionnaire. The questionnaires were self-completed, except where individuals were illiterate.

Results: Mean \pm SD age of the subjects was $41.2 \pm 7.9$ years and $93.4 \%$ of them were male. More than half of them used heroin. Prevalence of poor sleeping and sexual dysfunction in patients under MMT were $67.7 \%$ and $18.2 \%$, respectively. There was no association between sleep quality or sexual dysfunction and demographics or methadone dose. However, a significant correlation was observed between mental health and sleep quality $(\mathrm{r}=0.16, \mathrm{P}=0.033)$, and sexual function $(\mathrm{r}=0.18, \mathrm{P}=0.011)$.

Conclusions: Sleep quality showed a poorer profile than sexual function. Therefore, more emphasis should be laid on treatment of sleep disparity during follow up of MMT patients comparing to their sexual function. Patients should be reassured that probable sexual dysfunctions should not be regarded as a consequence of MMT.
\end{abstract}

Keywords: Methadone, Sexual Dysfunction, Opiate Substitution Treatment, Substance-Related Disorders

\section{Background}

Methadone maintenance treatment (MMT) is an effective therapeutic modality for opiate addiction (1) and has beneficial impact on the frequency of opioid use, mental health, and quality of life; also, it could reduce high risk and criminal behaviors (2). Currently, there are many opioid dependent individuals receiving methadone for the treatment of addiction worldwide (3). The success of MMT is not solely dependent on drug abstinence, but other aspects of opioid maintenance treatment such as the impact of MMT on sleep quality (4) and sexual function should be kept in mind (5). Based on our anecdotal findings, sleep disparity and sexual dysfunction are amongst the most important concerns of patients under MMT (2).

Good sleep quality is one of the prerequisites of an acceptable physical and mental health. Poor sleep quality has been shown to be associated with many ill health conditions such as obesity, diabetes, and hypertension (6). The reported proportion of patients under MMT, who complain of sleep disturbances are more than $75 \%$ (7). Several mechanisms have been implicated for the poor sleep quality among patients under MMT such as decrease in acetylcholine release, suppression of inhibitory GABAergic transmission, and reduction of the nucleoside adenosine in some brain regions (7). Sleep difficulties in patients under MMT impair their quality of life and may decrease methadone treatment adherence and accordingly predispose them to other illicit drug use to help with sleep (8). On the other hand, there are cross-cultural differences in sleep quality, and the majority of studies in sleep quality have been conducted in developed countries and less is known about the issue in developing world, especially in Eastern Mediterranean countries $(7,8)$. 
Sexual dysfunction is a common and important complaint among psychiatric patients and patients with MMT are no exception. Besides, it is noteworthy that one of the motives behind drug use is increasing sexual pleasure (9). Although several studies have examined the effect of drug use on sexual activity, their results are not in line with each other, which may be due to using nonvalidated questionnaires (10). Recent studies are in favor of impairment of all dimensions of sexual functioning (i.e., pleasure, drive, arousal, and orgasm) in drug users, which may not be temporary (11). However, there are not only a few studies addressed sexual function in MMT patients, but also the majority of them are related to male sex only $(5,10)$. The importance of research on sexual function of patients under MMT becomes more evident, when we notice that sexual dysfunction may lead to discontinuation of maintenance treatment (5). So examining the different aspects of sexual function of patients under MMT warrants more research considering both sexes.

\section{Objectives}

Owing to association of sleep disorders with sexual activity (12), this study aimed to assess the sleep quality and sexual function together in patients on methadone maintenance treatment and to explore the interrelationship between sexual function and sleep quality in a sample of Iranian patients. This study may be useful for cross-cultural comparisons too.

\section{Patients and Methods}

\subsection{Setting and Participants}

This cross-sectional study was conducted in 5 MMT clinics of Kerman city located in the southeastern part of Iran. Two hundred and ten patients were invited for interview based on systematic random sampling scheme. Inclusion criteria were being older than 18 years, having no major physical or mental disorder according to history and being under MMT for at least 6 months. All subjects were informed about the nature and objectives of the study and written informed consent was obtained before study enrollment. The protocol of the study was approved by Ethics Committee of Kerman Neuroscience Research Center.

\subsection{Measurement Tools}

A 3-part questionnaire was used. The first part consisted of Pittsburgh sleep quality index (PSQI), which assessed sleep quality and disturbances during the past 4 weeks (13). This 19-item self-rated questionnaire generates 7 component scores (subjective sleep quality, sleep latency, sleep duration, habitual sleep efficiency, sleep disturbance, sleeping medication use, and daytime dysfunction). Reliability and validity of the Persian version of PSQI has been well-established (14). A cutoff value above 5 indicated poor sleeping. The second part included Arizona sexual experience scale (ASEX) to assess sexual dysfunction. It is composed of 5 items rated on a 6-point Likert-type scale, with higher scores reflecting greater sexual dysfunction (15). Each item quantifies a major domain of sexual function; sexual drive, psychological arousal, physiologic arousal (penile erection/vaginal lubrication), ability to reach orgasm, and orgasm satisfaction. For each item a score $\geq 5$ or a total score $\geq$ 19 was considered as sexual dysfunction. The Persian version of ASEX has shown good psychometric properties (16). The third part of our measurement tool, the 12-item general health questionnaire (GHQ-12) was used to assess mental health of participants. A higher score indicated a higher degree of mental disorder and the Persian version of GHQ-12 has been well-established (17). The fourth part of questionnaire consisted of demographic and drug related variables such as age, sex, and methadone dosage. The respondents completed anonymously all questionnaires. For illiterate subjects, the questionnaire was filled out through interview.

\subsection{Statistical Analysis}

We used Chi-square test to compare frequency of categorical variables between two groups was used. Also, to examine correlation between GHQ-12, and PSQI and ASEX scores, Pearson correlation coefficient was used. P value less than 0.05 was considered significant.

\section{Results}

Out of 210 subjects invited for the interview, 198 participated in the study (response rate $=94.3 \%$ ). Mean \pm SD age of the participants was $41.2 \pm 7.9$ years. Most of them were male (93.4\%) and $4.5 \%$ were illiterate. Other demographic characteristics are shown in Table 1 . The most frequent substance used was heroin (54.5\%).

Prevalence of poor sleeping did not differ with respect to baseline characteristics except for marital status (Table 1). Frequency of sexual dysfunction was comparable between different subgroups of baseline variables. Overall, prevalence of poor sleeping and sexual dysfunction among patients under MMT were $67.7 \%$ and $18.2 \%$, respectively.

Mean scores of 7 components of PSQI are shown in Table 2. Considering the mean global PSQI score of general population $(4.71 \pm 2.11)(14)$, the score of patients under MMT was significantly lower than normal population ( $\mathrm{P}$ $<0.001$ ). Mean score of different aspects of sexual function of patients under MMT are shown in Table 2.

Only in men a significant correlation was seen between sleep quality and sexual function $(r=0.30, P<0.001)$, whilst in women no correlation was seen $(\mathrm{r}=-0.17, \mathrm{P}=$ $0.594)$. There was a significant correlation between mental health and sleep quality $(\mathrm{r}=0.16, \mathrm{P}=0.033)$, as well as sexual function $(\mathrm{r}=0.18, \mathrm{P}=0.011)$. 
Kheradmand $A$ et al.

Table 1. Baseline Characteristics of Patients Under Methadone Maintenance Treatment According to Sleep Quality and Sexual Quality ${ }^{\mathrm{a}}$

\begin{tabular}{|c|c|c|c|c|c|}
\hline \multirow{2}{*}{ Characteristics } & \multirow{2}{*}{$\mathbf{f}(\%)$} & \multicolumn{2}{|c|}{ Poor Sleepers } & \multicolumn{2}{|c|}{ Sexual Dysfunction } \\
\hline & & Values $^{\mathrm{b}}$ & PValue & Values $^{\mathrm{b}}$ & P Value \\
\hline Age, y & & & 0.578 & & 0.718 \\
\hline$<30$ & $16(8.1)$ & $9(56.2)$ & & $2(12.5)$ & \\
\hline $30-50$ & $155(78.3)$ & $107(69.0)$ & & $30(19.4)$ & \\
\hline$\geq 50$ & $27(13.6)$ & $18(66.7)$ & & $4(14.8)$ & \\
\hline Gender & & & 0.554 & & 0.709 \\
\hline Male & $185(93.4)$ & $124(67.0)$ & & $33(17.8)$ & \\
\hline Female & $13(6.6)$ & $10(76.9)$ & & $3(23.1)$ & \\
\hline Education & & & 0.682 & & 0.584 \\
\hline Illiterate & $9(4.5)$ & $5(55.6)$ & & $3(33.3)$ & \\
\hline Primary & $29(14.6)$ & $23(79.3)$ & & $3(10.3)$ & \\
\hline Secondary & $81(40.9)$ & $51(63.0)$ & & $15(18.5)$ & \\
\hline Diploma & $65(32.8)$ & $46(70.8)$ & & $13(20.0)$ & \\
\hline College & $14(7.1)$ & $9(64.3)$ & & $2(14.3)$ & \\
\hline Marital status & & & 0.037 & & 0.454 \\
\hline Married & $155(78.3)$ & $98(63.2)$ & & $26(16.8)$ & \\
\hline Single & $28(14.1)$ & $24(85.7)$ & & $5(17.9)$ & \\
\hline Divorced & $15(7.6)$ & $12(80.0)$ & & $5(33.3)$ & \\
\hline Job & & & 0.104 & & 0.051 \\
\hline Unemployed & $29(14.6)$ & $26(86.2)$ & & $2(6.9)$ & \\
\hline Part time & $137(69.2)$ & $95(69.3)$ & & $31(22.6)$ & \\
\hline Full time & $32(16.2)$ & $20(62.5)$ & & $3(9.4)$ & \\
\hline Main substance abused & & & 0.359 & & 0.161 \\
\hline Heroin & $108(54.5)$ & $82(75.9)$ & & $16(14.8)$ & \\
\hline Opium & $50(25.3)$ & $33(66)$ & & $8(16.0)$ & \\
\hline Opium residue & $35(17.7)$ & $22(62.9)$ & & $10(28.6)$ & \\
\hline $\begin{array}{l}\text { Crystalline methamphetamine } \\
\text { (Shisheh in Persian) }\end{array}$ & $5(2.5)$ & $3(60.0)$ & & $2(40.0)$ & \\
\hline Dose level & & & 0.338 & & 0.470 \\
\hline Low & $10(5.1)$ & $6(60.0)$ & & $1(10.0)$ & \\
\hline Medium & $27(13.6)$ & $22(81.5)$ & & $7(25.9)$ & \\
\hline High & $161(81.3)$ & $112(69.6)$ & & $28(17.4)$ & \\
\hline Total & & $134(67.7)$ & - & $36(18.2)$ & - \\
\hline
\end{tabular}

$\mathrm{a}_{\mathrm{N}=198 .}$

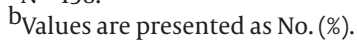


Kheradmand A et al.

\begin{tabular}{|c|c|}
\hline Items & Mean \pm SD \\
\hline \multicolumn{2}{|l|}{ PSQI component } \\
\hline Subjective sleep quality & $1.07 \pm 0.03$ \\
\hline Sleep latency & $1.05 \pm 0.05$ \\
\hline Sleep duration & $1.20 \pm 0.06$ \\
\hline Habitual sleep efficiency & $0.79 \pm 0.07$ \\
\hline Sleep disturbance & $1.05 \pm 0.02$ \\
\hline Sleeping medication use & $0.96 \pm 0.09$ \\
\hline Daytime dysfunction & $0.78 \pm 0.06$ \\
\hline PSQI total & $6.9 \pm 0.20$ \\
\hline \multicolumn{2}{|l|}{ ASEX items (men) } \\
\hline Sexual drive & $3.08 \pm 0.07$ \\
\hline Psychological arousal & $2.91 \pm 0.07$ \\
\hline Erection & $3.01 \pm 0.08$ \\
\hline Ease of orgasm & $2.89 \pm 0.08$ \\
\hline Orgasm satisfaction & $2.87 \pm 0.07$ \\
\hline Total & $14.70 \pm 0.31$ \\
\hline \multicolumn{2}{|l|}{ ASEX items (women) } \\
\hline Sexual drive & $3.23 \pm 0.17$ \\
\hline Psychological arousal & $3.46 \pm 0.35$ \\
\hline Vaginal lubrication & $2.92 \pm 0.26$ \\
\hline Ease of orgasm & $3.54 \pm 0.35$ \\
\hline Orgasm satisfaction & $3.62 \pm 0.27$ \\
\hline Total & $16.77 \pm 1.19$ \\
\hline
\end{tabular}

${ }^{a} \mathrm{~N}=198$.

\section{Discussion}

MMT is considered an effective therapeutic modality for opioid dependent patients, owing to both improving the quality of life and decreasing the HIV risk taking behaviors and criminal acts (2). Every effort to decrease attrition of patients under MMT is an important step in settlement of this harm reduction strategy. Poor sleep quality and sexual dysfunction are two potential threats to continuation of MMT and compliance of patients receiving this treatment modality in Western countries (7, $8,10,11)$. There is a paucity of studies addressing the issue from Non-Western world (18), and to the best of our knowledge, no study has been published in Eastern Mediterranean region. The main limitation of the study was the lack of control group to compare the findings with those of general population.

This study revealed that more than two-third of patients reported poor sleeping, whilst the frequency of sexual dysfunction was much lower (i.e. less than one-fifth). The main advantages of this study were an adequate sample size, inclusion of both sexes, and addressing sleep quality and sexual function together. The study limitation was lack of data to compare sexual function with general population.

The frequency of poor sleeping did not differ according to baseline criteria except for job status, in which unemployed subjects suffered more than employed ones from poor sleep. Job insecurity as one of the social determinants of health has been considered a contributing factor in sleep disparity (19). Therefore, asking about quality of sleep among unemployed subjects is very important. Other studies have shown no association between sleep quality and demographic characteristics such as age and sex in patients receiving MMT (8), which is in line with our findings. In our study, sleep quality was not related to methadone dosage. According to Stein et al. there is no association between methadone dosage and subjective sleep disturbances (7). However, in a Taiwanese study, an association was found between PSQI scores and methadone dosage (20). The quality of sleep in patients under MMT was poor both in terms of high percentage of poor sleepers and in terms of comparison of mean PSQI score of them with general population. 
Sexual dysfunction was seen in $18.2 \%$ of subjects which was lower than some other relevant studies $(5,10)$. The prevalence of sexual function among patients under MMT remains a controversial issue. Some reports are in favor of a higher percentage than general population (3) and some state that the overall prevalence of sexual dysfunction in these patients is "similar to general population" (i.e. between 5\% and 35\%) (5). The low percentage in our study may be due to the exclusion of patients with history of physical and or psychiatric disorders. Medical conditions and mental disorders have a causal role in sexual dysfunction (5). Prevalence of sexual dysfunction was not significantly different between subgroups of patients. Hanbury et al. found no relationship between sexual dysfunction and demographics or dose of methadone in patients enrolled in a methadone maintenance program (21). Lack of association between methadone dose and sexual dysfunction was reported by Brown et al. too (5). So unlike sleep disparity, sexual dysfunction may not be a major concern in patients under MMT. There was a significant correlation between PSQI score and ASEX score only in men. This means that in men, the higher the sexual dysfunction, the poorer would be the sleep quality. Due to different coping styles in sexual dysfunction between two sexes (18), gender differences should be mentioned while assessing sexual function as well as sleep quality in patients receiving MMT.

Overall, this study showed that sleep quality was poor in the majority of patients under MMT for more than 6 months. Sexual dysfunction may not be a major concern during history taking about methadone side effects, but gender differences should be kept in mind during patients' follow up.

\section{Acknowledgments}

We acknowledge financial support of Kerman Neuroscience Research Center.

\section{Footnotes}

Authors' Contribution:Drafting of the manuscript and statistical analysis: Nouzar Nakhaee; data collection: Zahra Zeynali and Zahra Amini-Ranjbar; conducting the study and finalizing the proposal: Ali Kheradmand and Abdol-Reza Sabahy.

Funding/Support:Kerman Neuroscience Research Center.

\section{References}

1. Wu Z, Clark N. Scaling up opioid dependence treatment in low- and middle-income settings. Bull World Health Organ. 2013;91(2):82-82A. doi:10.2471/BLT.12.110783. [PubMed: 23554516]

2. Esmaeili HR, Ziaddinni H, Nikravesh MR, Baneshi MR, Nakhaee $\mathrm{N}$. Outcome evaluation of the opioid agonist maintenance treatment in Iran. Drug Alcohol Rev. 2014;33(2):186-93. doi:10.1111/ dar.12112. [PubMed: 24428135]

3. Brown RT, Zueldorff M. Opioid substitution with methadone and buprenorphine: sexual dysfunction as a side effect of therapy. Heroin Addict Relat Clin Probl. 2007;9(1):35-44.

4. Pud D, Zlotnick C, Lawental E. Pain depression and sleep disorders among methadone maintenance treatment patients. Addict Behav. 2012;37(11):1205-10. doi: 10.1016/j.addbeh.2012.05.025. [PubMed: 22742985]

5. Brown R, Balousek S, Mundt M, Fleming M. Methadone maintenance and male sexual dysfunction. J Addict Dis. 2005;24(2):91106. doi:10.1300/J069v24n02_08. [PubMed:15784526]

6. Knutson KL. Sociodemographic and cultural determinants of sleep deficiency: implications for cardiometabolic disease risk. Soc Sci Med. 2013;79:7-15. doi: 10.1016/j.socscimed.2012.05.002. [PubMed: 22682665]

7. Stein MD, Kurth ME, Sharkey KM, Anderson BJ, Corso RP, Millman $\mathrm{RP}$. Trazodone for sleep disturbance during methadone maintenance: a double-blind, placebo-controlled trial. Drug Alcohol Depend. 2012;120(1-3):65-73. doi: 10.1016/j.drugalcdep.2011.06.026. [PubMed: 21798674]

8. Peles E, Schreiber S, Adelson M. Variables associated with perceived sleep disorders in methadone maintenance treatment (MMT) patients. Drug Alcohol Depend. 2006;82(2):103-10. doi: 10.1016/j.drugalcdep.2005.08.011. [PubMed:16154297]

9. Nakhaee N, Jadidi N. Why do some teens turn to drugs? a focus group study of drug users' experiences. J Addict Nurs. 2009;20(4):203-8. doi:10.3109/10884600903291158.

10. Hallinan R, Byrne A, Agho K, McMahon C, Tynan P, Attia J. Erectile dysfunction in men receiving methadone and buprenorphine maintenance treatment. J Sex Med. 2008;5(3):684-92. doi: 10.1111/j.1743-6109.2007.00702.x. [PubMed: 18093096]

11. Vallejo-Medina P, Sierra JC. Effect of drug use and influence of abstinence on sexual functioning in a Spanish male drug-dependent sample: a multisite study. J Sex Med. 2013;10(2):333-41. doi: 10.1111/j.1743-6109.2012.02977.x. [PubMed:23095213]

12. Schenck CH, Arnulf I, Mahowald MW. Sleep and sex: what can go wrong? A review of the literature on sleep related disorders and abnormal sexual behaviors and experiences. Sleep. 2007;30(6):683-702. [PubMed:17580590]

13. Buysse DJ, Reynolds CF, Monk TH, Berman SR, Kupfer DJ. The Pittsburgh Sleep Quality Index: a new instrument for psychiatric practice and research. Psychiatry Res. 1989;28(2):193-213. [PubMed: 2748771]

14. Farrahi Moghaddam J, Nakhaee N, Sheibani V, Garrusi B, Amirkafi A. Reliability and validity of the Persian version of the Pittsburgh Sleep Quality Index (PSQI-P). Sleep Breath. 2012;16(1):79-82. doi: 10.1007/s11325-010-0478-5. [PubMed: 21614577]

15. McGahuey CA, Gelenberg AJ, Laukes CA, Moreno FA, Delgado PL, McKnight KM, et al. The Arizona Sexual Experience Scale (ASEX): reliability and validity. J Sex Marital Ther. 2000;26(1):25-40. [PubMed:10693114]

16. Pezehki MZ, Bayrami R, editors. Reliability and construct validity of Arizona Sexual Experience Scale (ASEX) among pregnant women referred to Tabriz urban health centers, 2004.; 2nd national congress on family and sexual problems.; 2005; Tehran. p. 131.

17. Montazeri A, Harirchi AM, Shariati M, Garmaroudi G, Ebad M, Fateh A. The 12-item General Health Questionnaire (GHQ12): translation and validation study of the Iranian version. Health Qual Life Outcomes. 2003;1:66. doi: 10.1186/1477-7525-1-66. [PubMed: 14614778]

18. Xia Y, Zhang D, Li X, Chen W, He Q, Jahn HJ, et al. Sexual dysfunction during methadone maintenance treatment and its influence on patient's life and treatment: a qualitative study in South China. Psychol Health Med. 2013;18(3):321-9. doi: 10.1080/13548506.2012.729845. [PubMed: 23092392]

19. Grunstein RR. Global perspectives on sleep and health issues. $J$ Natl Inst Public Health. 2012;61(35):e42.

20. Hsu WY, Chiu NY, Liu JT, Wang CH, Chang TG, Liao YC, et al. Sleep quality in heroin addicts under methadone maintenance treatment. Acta Neuropsychiatr. 2012;24(6):356-60. doi: 10.1111/j.16015215.2011.00628.x. [PubMed: 25287178]

21. Hanbury R, Cohen M, Stimmel B. Adequacy of sexual performance in men maintained on methadone. Am J Drug Alcohol Abuse. 1977;4(1):13-20. [PubMed: 565588] 